\title{
Three Synchronous Tumors Discovered in SIADH Investigation
}

\author{
Ricardo Fonseca, Filipa Monteiro, Rita Mendes \\ Internal Medicine Department, Hospital Egas Moniz, Centro Hospitalar Lisboa Ocidental, Lisbon, Portugal
}

How to cite this article: Fonseca R, Monterio F, Mendes R. Three synchronous tumors discovered in SIADH investigation. EJCRIM 2017;4: doi:10.12890/2017_000594.

Conflicts of Interests: The Authors declare that there are no competing interests.

This article is licensed under a Commons Attribution Non-Commercial 4.0 License

\section{ABSTRACT}

Syndrome of Inappropriate Antidiuretic Hormone Secretion (SIADH) is a common cause of euvolemic hyponatremia which has many possible etiologies such as tumors (mainly lung cancers). We present a case of a 79-year old women hospitalized due to microcytic anemia with transfusion need and hyponatremia due to SIADH. Three primary tumors without known metastasis were discovered. The case shows the importance of studying causes of hyponatremia.

\section{LEARNING POINTS}

- Hyponatremia is a common electrolytic disorder during hospitalization and is associated with greater mortality.

- Correction of hyponatremia, cause can be devastating unless the underlying causes are investigated as it can be caused by severe diseases.

- Cancer must be excluded in the absence of an obvious etiology of SIADH (drugs, pulmonary and nervous system disorders).

\section{KEYWORDS}

Hyponatremia, SIADH, Synchronous tumors

\section{INTRODUCTION}

SIADH is a major cause of hypotonic hyponatremia with normal extracellular fluid volume and is characterized by hyponatremia, plasma osmolality $<275 \mathrm{mOsm} / \mathrm{kg}$, urine osmolality $>100 \mathrm{mOsm} / \mathrm{kg}$, urine sodium concentration $>30 \mathrm{mmol} / \mathrm{l}$, and low serum urea and uric acid in the absence of other causes (diuretics, adrenal and thyroid insufficiency) ${ }^{[1]}$. It could be due to malignant diseases, drugs, or pulmonary or nervous system disorders ${ }^{[2]}$. Tumoral hyponatremia is more common in small-cell lung cancer than other solid and hematological malignancies ${ }^{[3]}$. Besides some study heterogeneity, hyponatremia seems to be a negative prognostic factor in cancer patients ${ }^{[4]}$. Management of hyponatremia includes fluid restriction, saline infusion, and/or drugs (furosemide, urea and vasopressin receptor antagonists) ${ }^{[5]}$.

\section{CASE REPORT}

This case is a 79-years old Caucasian woman with a history of hypertension (treated with irbesartan), type 2 diabetes (treated with metformin, glimepiride, and sitaglitin) and microcytic anemia hospitalized for hyponatremia (119nmol/L) and worsening anemia with red cell concentrates transfusion need $(\mathrm{Hb} 5.7 \mathrm{~g} / \mathrm{dL})$. The patient had asthenia, anorexia, and dizziness, and denied nausea, vomiting, melena, hematochezia, metrorrhagia, excessive water ingestion, and changes in weight.

On admission, patient had pale skin, tachycardia (110 bpm), pulmonary auscultation was normal, her abdomen was soft and without palpable masses or organomegaly. She did not have dry skin or edema. 
Initial blood test results: hemoglobin $5.7 \mathrm{mg} / \mathrm{dL}$ (12-15), mean corpuscular volume 67.1fL (80-96), mean corpuscular hemoglobin 25.3pg (27.3-37.3), platelets 515×109/L (150-400), serum creatinine $0.77 \mathrm{mg} / \mathrm{dL}$ (0.5-0.9), sodium 119mmol/L (136-145), chloride 88mmol/L (98107), potassium 5.1 mmol/L (3.5-5.1), normal transaminases, c-reactive protein $9 \mathrm{mg} / \mathrm{dL}(<0.5)$. The hyponatremia study is shown in Table 1.

\begin{tabular}{|l|c|c|}
\hline & Patient & Reference range \\
\hline Creatinine (mg/dL) & 0.77 & $0.5-0.9$ \\
\hline $\mathrm{Na}+(\mathrm{mmol} / \mathrm{L})$ & 145 & $135-145$ \\
\hline $\mathrm{K}+(\mathrm{mmol} / \mathrm{L})$ & 3,92 & $3,5-5,2$ \\
\hline Plasma osmolality (mOsmol/L) & 260 & $275-295$ \\
\hline Urinary osmolality (mOsmol/L) & 346 & $300-900$ \\
\hline Urinary sodium (mmol/L) & 88 & $40-220$ \\
\hline Morning serum cortisol (mcg/dL) & 32.3 & $6.2-19.4$ \\
\hline TSH (uU/mL) & 2.23 & $0,4-4$ \\
\hline Uric acid (mg/dL) & 3.0 & $2.4-5.7$ \\
\hline Glucose (mg/dL) & 214 & $74-106$ \\
\hline
\end{tabular}

Table 1. Hyponatremia study

Euvolemic hyponatremia was diagnosed. After exclusion of hypothyroidism, suprarenal insufficiency, and in the absence of diuretics, the relatively high urinary osmolality $(>300)$ and sodium $(>30)$ for the level of serum sodium and plasmatic osmolality confirmed the diagnosis of SIADH but there was no clear etiology. None of the drugs the patient used were linked to SIADH. A gastrointestinal tract tumor was suspected due to microcytic anemia so an endoscopy and a colonoscopy were planned. Meanwhile, chest radiograph revealed a heterogeneous hypotransparency in the right hemithorax medium third (Fig. 1). A chest tomography was performed to clarify this finding which showed a single right lung mass, suggesting primary cancer. Tumoral marker tests were within reference range (CA $12.913 \mathrm{U} / \mathrm{mL}$, Neuron-specific enolase $7.8 \mathrm{ng} / \mathrm{mL}$, squamous cell carcinoma antigen $0.9 \mathrm{ng} / \mathrm{mL}, \mathrm{CEA} 4.3 \mathrm{ng} / \mathrm{mL}$ ).

In spite of the strongly suspected lung cancer, which is a common tumoral cause of SIADH, it was decided to continue the investigation because the cause of the microcytic anemia was as yet unclear. Endoscopy showed a gastric mass. Colonoscopy was also performed and revealed a cecal mass (Fig. 2), suggesting primary colon tumor. A biopsy by fibrobronchoscopy confirmed a poor differentiated lung adenocarcinoma, also suggesting primary tumor. Therefore, three possible primary tumors were discovered in the SIADH investigation. Patient had a good performance status so surgery was planned (divided in two phases, first colon plus stomach, then pulmonary).

During the extensive workup, the patient had fluid restriction and started furosemide, but symptomatic hyponatremia sustained. She started oral therapy of urea $30 \mathrm{~g} /$ day. Sodium remained stable within reference range until surgery. The evolution of sodium after hospitalization is shown in Figure 3.

Abdominal surgery composed of right hemicolectomy and partial gastrectomy was performed 4 weeks after initial hospitalization. Histopathological study confirmed GIST tumor (T2) and cecum adenocarcinoma (pT3, N1c). The patient died fifteen days after surgery due to a hospital infection before the final surgery could be carried out.

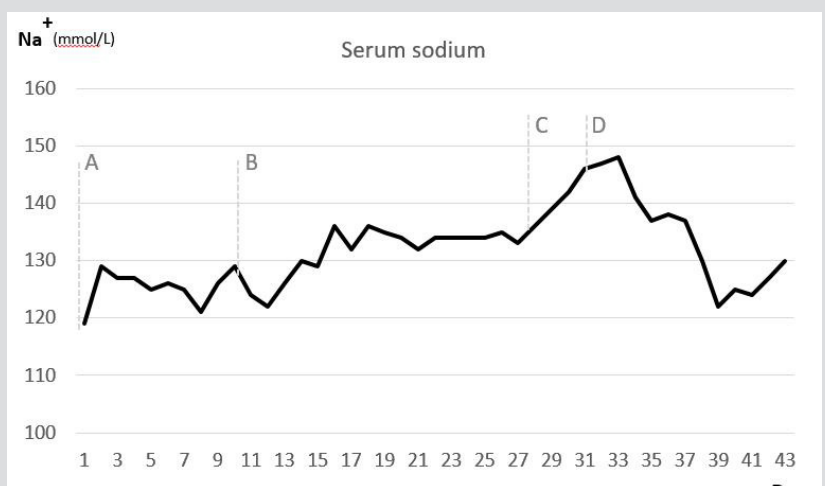

Davs
Figure 1. Serum sodium (mmol/L) evolution during hospitalization. Serum sodium (mmol/L) evolution during hospitalization. (A) Patient started fluid re-striction and furosemide; (B) Urea 30g/day was initiated; (C) Surgery hemicolectomy and partial gastrectomy; (D) Urea was stopped in the intensive care due to hypernatremia. 


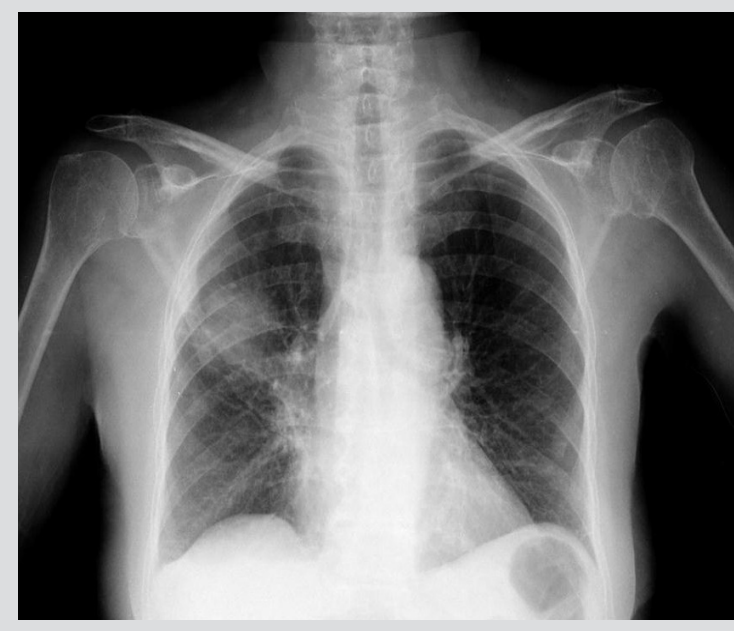

Figure 2. Right lung hypotransparency in chest radiography

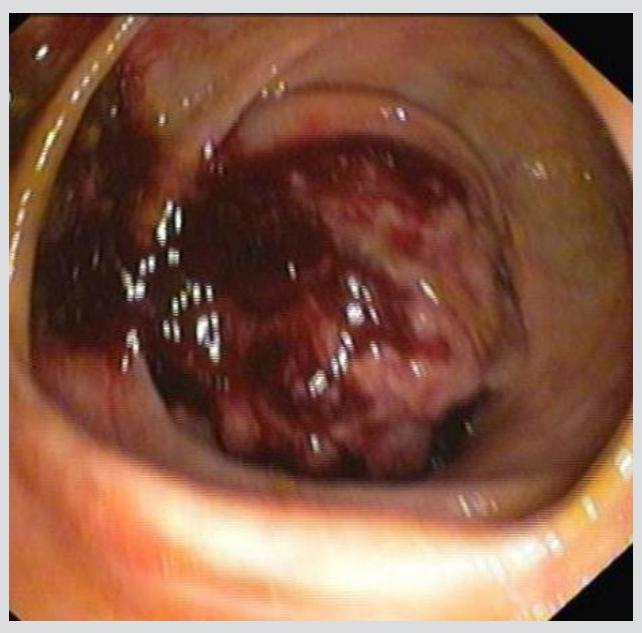

Figure 3. Colon tumor in colonoscopy

\section{DISCUSSION}

A case of euvolemic hyponatremia caused by SIADH is presented. Fluid restriction and furosemide were used to control sodium before treating the primary cause. It was not sufficient so urea $30 \mathrm{~g} /$ day with normalization of sodium was started. Extensive workup was needed to clarify the SIADH etiology. Lung tumors, particularly small-cell lung cancer are the most common causes of tumoral SIADH. A lung mass strongly suspected of primary lung tumor was initially found. Normally this would be enough to complete the SIADH study. However, it did not explain the microcytic anemia with transfusion need. A gastrointestinal tumor due to microcytic anemia was suspected from the start. There could also be another gastrointestinal cause of anemia due to blood loss, potentially treatable (such as peptic ulcer bleeding, angiodysplasia, or bleeding colon polyp), so it was decided to continue the investigation finding GIST and colon carcinoma. This would not have been done in the absence of severe microcytic anemia.

Consequently, this was an unusual setting of three primary tumors without known metastasis confirmed in histopathological study. It is difficult to say which tumor was causing the SIADH so possibly more than one. However, , besides the limitation of surgery losses and posterior fluid management, analyzing the evolution of sodium shows that SIADH was not resolved so the lung tumor could still have produced antidiuretic hormone.

\section{REFERENCES}

1. Spasovski G, Vanholder R, Allolio B, Annane D, Ball S et al. Clinical practice guideline on diagnosis and treatment of hyponatraemia. Eur J Endocrinol 2014;170:G1-G47.

2. Liamis G, Milionis H. Elisaf M. Causes of the syndrome of inappropriate antidiuresis. Am J Kidney Dis 2008;52:144-153.

3. Castillo JJ, Vincent M, Justice E. Diagnosis and management of hyponatremia in cancer patients. Oncologist 2012;17:756-65.

4. Berardi R, Rinaldi S, Caramanti M, Grohè C, Santoni M et al. Hyponatremia in cancer patients: Time for a new approach. Crit Rev Oncol Hematol 2016;102:15-25.

5. Ball SG, Iqbal Z. Diagnosis and treatment of hyponatraemia. Best Pract Res Clin Endocrinol Metab 2016;30:161-73. 